Preprint MCGILL-97/36

e-Print Archive: hep-ph/9801204

\title{
A Nonperturbative Measurement of the Broken Phase Sphaleron Rate
}

\author{
Guy D. Moore' \\ Dept. of Physics, McGill University \\ 3600 University St. \\ Montreal, PQ H3A 2T8 Canada
}

\begin{abstract}
We develop a method to compute the sphaleron rate in the electroweak broken phase nonperturbatively. The rate is somewhat slower than a perturbative estimate. In $\mathrm{SU}(2) \times \mathrm{U}(1)$ Higgs theory at the physical value of $\Theta_{W}$, and assuming that the latent heat of the phase transition reheats the universe to the equilibrium temperature, baryon number erasure after the phase transition is prevented only when $\left(\lambda / g^{2}\right) \leq 0.036$.
\end{abstract}

PACS numbers: 11.15.Ha, 11.15.Kc

Key words: sphaleron, baryogenesis, baryon number violation, electroweak phase transition, lattice gauge theory

\section{Introduction}

In the past ten years the effort to understand electroweak baryogenesis has driven the study of the physics of the hot electroweak plasma at and around the electroweak phase transition. The relevant physics has been put on a much sounder foundation, and in particular we now possess reliable nonperturbative studies of the strength of the phase transition and of its disappearance at sufficiently high scalar (Higgs) self-coupling [1, 2, 3].

Electroweak baryogenesis in the minimal standard model is now ruled out on two independent grounds. First, it cannot produce enough baryons because there is not enough CP violation in the minimal standard model [4]. Second, even if it could produce the baryons, they would be wiped out in the aftermath of the phase transition, because the rate of baryon number violating processes does not shut off fast enough after the phase transition. This is because it is excluded that the standard model Higgs boson can have a weak enough selfcoupling to supply the required strength to the phase transition [1]. In fact, experimental bounds on the Higgs mass are now high enough that if the minimal standard model is the right physical theory, we can conclude that there was no cosmological electroweak phase transition.

Extensions of the standard model, such as the minimal supersymmetric standard model with a light stop, appear to be alive and kicking for at least part of their parameter space.

${ }^{1}$ e-mail: guymoore@physics.mcgill.ca 


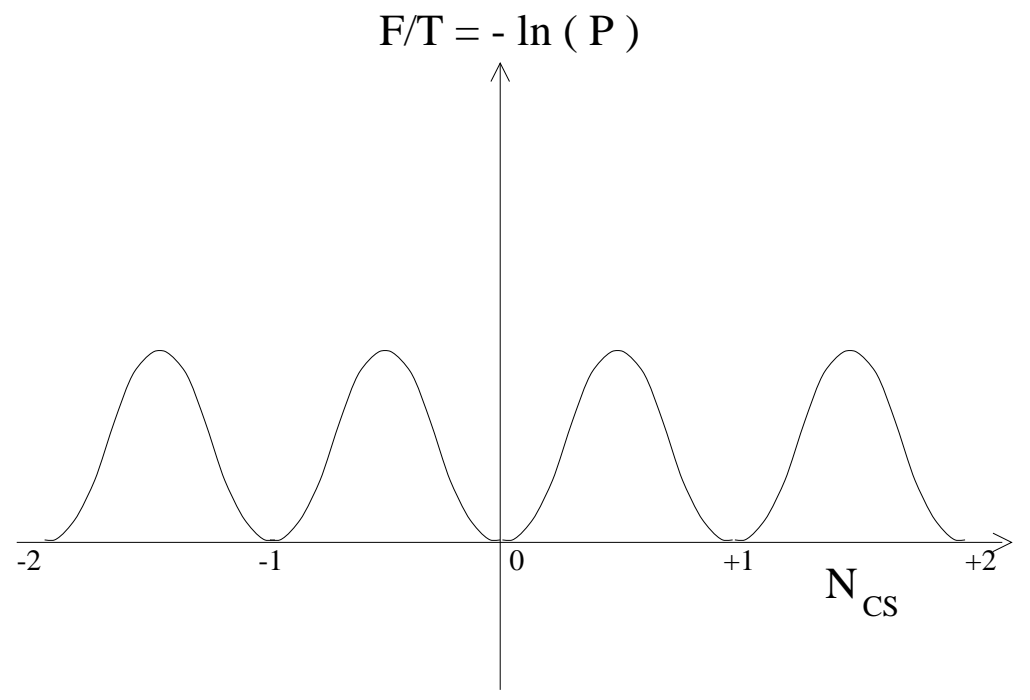

Figure 1: "Cartoon" of the free energy dependence on $N_{\mathrm{CS}}$.

To determine what parts of their parameter space are viable, we demand that they pass two tests. There must be enough $\mathrm{CP}$ violation to produce sufficient baryon number to explain astrophysical observations; and baryon number must not be subsequently erased. The production depends on where CP violation appears in the theory and on the mechanism by which it causes a baryon number surplus. We will not address this question here. The erasure depends on the efficiency of baryon number violating processes after the phase transition. This paper will study this erasure nonperturbatively.

Arnold and McLerran established the basic picture for baryon number violation in the electroweak broken phase [5]. Baryon number change in the electroweak theory is proportional to change in the Chern-Simons number $\left(N_{\mathrm{CS}}\right)$ of the $\mathrm{SU}(2)$ fields, because of the anomaly [6]. But in the broken phase, there is a barrier to changing $N_{\mathrm{CS}}$. To change from one integer value to another, one must pass through a half integer state, and while there are configurations of integer $N_{\mathrm{CS}}$ with arbitrarily small energy, the energy of a configuration of half integer $N_{\mathrm{CS}}$ is bounded by the energy of Klinkhamer and Manton's "sphaleron" configuration [7]. At finite temperature the same statements are true for free energy, and one can use Langer's formalism [8, 9] to find the rate of baryon number violation. The classic cartoon of free energy as a function of $N_{\mathrm{CS}}$ is shown in Fig. 1. The hard part of getting from, say, $N_{\mathrm{CS}}=0$ to $N_{\mathrm{CS}}=1$ is getting over the peak (the sphaleron barrier) in between. Let us assume that, whenever the system passes over the peak, it subsequently gets stuck in the new minimum. Then the diffusion constant for $N_{\mathrm{CS}}$ is set by the flux of states in the thermal ensemble going over the barrier. The diffusion constant for $N_{\mathrm{CS}}$,

$$
\gamma_{d} \equiv \lim _{t \rightarrow \infty} \frac{\left\langle\left(N_{\mathrm{CS}}(t)-N_{\mathrm{CS}}(0)\right)^{2}\right\rangle}{t}
$$

is the probability per unit time of crossing the peak, which is the flux of states in the thermal ensemble over the peak. The flux of states over the peak is the probability to have $N_{\mathrm{CS}}$ within $\epsilon / 2$ of the peak, times the mean value of $\left|d N_{\mathrm{CS}} / d t\right|$ measured at the peak, divided by $\epsilon$. Since the diffusion constant should be extensive, we generally refer to the diffusion constant per 
unit volume, $\Gamma_{d} \equiv \gamma_{d} / V$, often called the sphaleron rate.

The height of the sphaleron barrier in the immediate aftermath of the electroweak phase transition depends on the broken phase expectation value of the Higgs condensate, $\phi \equiv$ $\sqrt{\left\langle\phi^{2}\right\rangle}$, at the ambient temperature. In the minimal standard model the latent heat of the transition is not quite enough to heat the plasma back to the equilibrium temperature, but in supersymmetric extensions, it may be; so we need the jump in $\left\langle\phi^{2}\right\rangle$ at $T_{\mathrm{c}}$. We now have reliable nonperturbative studies of this quantity [1, 3]. These studies were motivated largely so the determined value of $\left\langle\phi^{2}\right\rangle$ could be plugged into the calculation of the sphaleron rate. However, the present state of the art in the sphaleron rate is a one loop calculation. At the equilibrium temperature, we know that perturbation theory for infrared quantities such as $\Gamma_{d}$ is at best an expansion in $\lambda / g^{2}$, and we know of no reason why the expansion for $\Gamma_{d}$ should be better behaved than the expansion for $\left\langle\phi^{2}\right\rangle$. At tree level, the phase transition is second order, and $\Gamma_{d}$ is large just below $T_{\mathrm{c}}$ [5]. At one loop, the phase transition becomes first order and $\Gamma_{d}$ is exponentially suppressed up to $T_{\mathrm{c}}$. To see how well perturbation theory converges, we would like a two loop calculation. Our experience from the calculation of $\left\langle\phi^{2}\right\rangle$ is that two loop corrections may not be small.

Unfortunately, extending the perturbative calculation of $\Gamma_{d}$ to two loops is immensely harder than it is for $\left\langle\phi^{2}\right\rangle$. Both are background field calculations, but the background field in the effective potential calculation is spatially uniform, and the diagrams can be computed using Fourier techniques. The sphaleron background, in contrast, lacks translation symmetry, and is only known numerically. The one loop calculation demands summing the eigenvalues of numerically determined fluctuation eigenmodes in this background. At two loops one needs to compute mutual interactions between the fluctuations, which will involve double and triple sums over the eigenmodes of overlap integrals; and the interaction Hamiltonian depends on the numerically determined sphaleron background. In our opinion it is actually easier to make a direct nonperturbative calculation of $\Gamma_{d}$ than to perform this two loop calculation.

In the symmetric phase we can now determine $\Gamma_{d}$ with fair reliability, using real time techniques [10, 11, 12] too small for a transition to occur in a reasonable amount of Hamiltonian time. But if we knew what we meant by the horizontal axis in Figure 1, then we could use nonperturbative lattice tools to find the diffusion constant. Both the probability to be within $\epsilon / 2$ of the peak, and the instantaneous mean value of $\left|d N_{\mathrm{CS}} / d t\right|$, are thermodynamic properties, and while the probability to be near the peak is exponentially small, we could measure it using multicanonical Monte-Carlo techniques. That would give $\Gamma_{d}$ up to an order unity dynamical prefactor, reflecting the fact that when the system crosses the peak, it does not necessarily get stuck in the new phase, but may turn around and re-cross immediately. In the broken phase we expect this dynamical prefactor to be on order 1 ; we will estimate it in the conclusion.

Our approach in this paper is precisely to find a way to make sense of Fig. 1 in a nonperturbative lattice study of the dimensionally reduced electroweak theory, and to construct the

\footnotetext{
${ }^{2}$ Recently it has been pointed out [14], correctly, that in the parametric limit of small $\alpha_{w}$ there are logarithmic corrections to the functional form assumed for fitting in [12. The numerical value of the coefficient of the $\log$ can be computed in a simple effective theory [14], and the result is that the coefficient of the $\log$ is small [15]; so while there is an uncontrolled systematic in the most recent results for the symmetric phase rate [12], the error caused is relatively small.
} 
figure and find the probability to be at the peak by multicanonical Monte-Carlo techniques. We also need to measure $\left\langle\left|d N_{\mathrm{CS}} / d t\right|\right\rangle$ at the peak, meaning the value averaged over the ensemble restricted to configurations with $N_{\mathrm{CS}}=1 / 2$. This paper will present the crux of the technique, with the essential ideas; but we will postpone some technical details to a longer sequel [13]. The outline of the paper is as follows. First we construct a suitable definition of $N_{\mathrm{CS}}$. Then we use it to perform a multicanonical Monte-Carlo determination of $\Gamma_{d}$. We compare our results at three values of $x \equiv \lambda / g^{2}$ to results of a perturbative calculation of $\Gamma_{d}$ made using the two loop effective potential. We determine that, in the minimal standard model, or any theory where heavy degrees of freedom can be integrated out until it looks like the minimal standard model [16], the sphaleron bound at the physical value of the Weinberg angle is $x \equiv \lambda / g^{2} \simeq 0.036$.

\section{$2 \quad$ Definition of $N_{\mathrm{CS}}$}

We will treat the thermodynamics of the hot standard model in the dimensional reduction approximation, so the configuration space and probability distribution are the same as thermal, classical Yang-Mills theory with certain Higgs mass counterterms. We use notation and field normalizations appropriate for the $3+1$ dimensional classical field theory and will determine the instantaneous value of $\left|d N_{\mathrm{CS}} / d t\right|$ in the classical theory context. But we need an appropriate definition of $N_{\mathrm{CS}}$.

Two features are essential to the definition of $N_{\mathrm{CS}}$ :

1. $N_{\mathrm{CS}}$ is the integral of the total derivative $\left(g^{2} / 8 \pi^{2}\right) E_{i}^{a} B_{i}^{a}$; and

2. $N_{\mathrm{CS}}$ is an integer for a vacuum configuration, $B_{i}^{a}=0$.

To determine the rate $\Gamma_{d}$ by constructing Fig. 11 we only need to define $N_{\mathrm{CS}}$ modulo 1 ; so we can set $N_{\mathrm{CS}}=0$ for all vacua, and we may hope for a gauge invariant definition.

To measure $N_{\mathrm{CS}}$ of some three dimensional gauge field configuration we then find a path from that configuration to a vacuum and integrate $E_{i}^{a} B_{i}^{a}$ along that path. The most natural path to choose is the cooling path; following [11] we define a cooling or dissipative time $\tau$, under which gauge fields evolve by dissipative dynamics,

$$
\frac{d A_{i}^{a}(x, \tau)}{d \tau}=-\frac{\partial H_{\mathrm{YM}}(A(\tau))}{\partial A_{i}^{a}(x, \tau)}, \quad A_{i}^{a}(x, 0)=A_{i}^{a}(x) .
$$

We write in continuum notation for clarity; the covariant lattice implementation is in [11], and Hetrick and de Forcrand have used a roughly equivalent procedure to solve the Gribov gauge fixing problem [17]. We cool only the gauge fields, under the Yang-Mills Hamiltonian, since $N_{\mathrm{CS}}$ should be a function of gauge fields alone; not cooling the Higgs fields also avoids some technical problems.

In a finite volume, the gauge fields will cool towards a vacuum off a measure zero subspace of configurations which cool towards saddlepoints. Hence we can define $N_{\mathrm{CS}}$ as

$$
N_{\mathrm{CS}}=\frac{g^{2}}{8 \pi^{2}} \int_{\tau_{0}}^{\infty} d \tau \int d^{3} x E_{i}^{a}(x, \tau) B_{i}^{a}(x, \tau)
$$


where $E_{i}^{a}(\tau)$ is the field strength in the $i, \tau$ direction. The standard lattice implementation of $E_{i}^{a} B_{i}^{a}$ [18] is not a total derivative [19], but it is to good approximation for smooth fields, by which we mean fields with almost no UV excitation. Cooling removes UV excitations much more efficiently than IR ones-in a linear theory the amplitude of a mode decays as $\exp \left(-\tau k^{2}\right)$-so the shortcomings of the definition of $E_{i}^{a} B_{i}^{a}$, and regulation sensitivity more generally, go away at a very small value of $\tau$, and are not a problem. Also note that the cooling procedure and the definition of $E_{i}^{a} B_{i}^{a}$ are gauge invariant, and so is our definition of $N_{\mathrm{CS}}$.

We cut off the small $\tau$ part of the integration to remove UV contributions to $N_{\mathrm{CS}}$ which are unrelated to winding number change. In the continuum abelian theory, where

$$
N_{\mathrm{CS}}=\frac{g^{2}}{32 \pi^{2}} \int d^{3} x \epsilon_{i j k} F_{i j} A_{k}
$$

the mean square value of $N_{\mathrm{CS}}$ we would get if we used $\tau_{0}=0$ is

$$
\left\langle N_{\mathrm{CS}}^{2}\right\rangle=\frac{g^{4}}{1024 \pi^{4}} \int d^{3} x d^{3} y \epsilon_{i j k} \epsilon_{l m n}\left\langle F_{i j}(x) A_{k}(x) F_{l m}(y) A_{n}(y)\right\rangle
$$

Using Wick's theorem and the momentum representation of the propagator, in Feynman gauge, this becomes

$$
\begin{aligned}
\left\langle N_{\mathrm{CS}}^{2}\right\rangle & =\frac{g^{4}}{1024 \pi^{4}} \int d^{3} x d^{3} y \int \frac{d^{3} p d^{3} q}{(2 \pi)^{6}} e^{i(p+q) \cdot(x-y)} \epsilon_{i j k} \epsilon_{l m n}\left(\frac{4 p_{i} p_{l} T^{2} \delta_{j m} \delta_{k n}}{p^{2} q^{2}}+\frac{4 p_{i} q_{l} T^{2} \delta_{j n} \delta_{k m}}{p^{2} q^{2}}\right) \\
& =\frac{g^{4} T^{2} V}{64 \pi^{4}} \int \frac{d^{3} p}{(2 \pi)^{3}} \frac{p^{2}}{\left(p^{2}\right)^{2}} .
\end{aligned}
$$

so $N_{\mathrm{CS}}$ will be Gaussian distributed with a linearly divergent variance. On the lattice, the UV divergence will be cut off by the lattice scale; the coefficient was found by Amjorn and Krasnitz [18 and is $\left\langle N_{\mathrm{CS}}^{2}\right\rangle=\left(1.44 \times 10^{-5}\right) g^{4} V T^{2} / a$.

$\mathrm{SU}(2)$ theory will have similar UV contributions, since in the UV it looks like three copies of the abelian theory. This UV contribution has nothing do with topology, since it appears already in the abelian theory. If we used $\tau_{0}=0$ then the measured value of $N_{\mathrm{CS}}$ would be an "interesting" IR piece plus this nontopological UV piece. The probability distribution of $N_{\mathrm{CS}}$ would be the convolution of the probability distributions for the two, and if the noise distribution were broad, this would spread $N_{\mathrm{CS}}^{\mathrm{IR}} \simeq 0$ configurations out to dominate the sample at $N_{\mathrm{CS}}=1 / 2$, destroying the peak in Fig. 1. Only if the noise distribution is narrow will the probability distribution be undistorted.

At finite $\tau_{0}$, and including the mass for the gauge fields which appears in the broken phase, the UV contribution to $N_{\mathrm{CS}}$, Eq. (6), becomes approximately

$$
\left\langle N_{\mathrm{CS}}^{2}\right\rangle \simeq \frac{g^{4} T^{2} V}{64 \pi^{4}} \int \frac{d^{3} p}{(2 \pi)^{3}} \frac{p^{2} e^{-4 p^{2} \tau_{0}}}{\left(p^{2}+m_{W}^{2}\right)^{2}} .
$$

(Note that $\tau$ has units of length squared, not length.) Choosing $\tau_{0} \geq 1 / m_{W}^{2}$ controls the noise sufficiently, while leaving $N_{\mathrm{CS}}$ with the feature which is essential to our endeavor, namely that a configuration which is very nearly a sphaleron (meaning that it cools to very close to 
the "sphaleron" saddlepoint, but then slips off and finds a vacuum) will have $N_{\mathrm{CS}}$ very close to $\pm 1 / 2$. We have checked that the part of the cooling path from (almost) the sphaleron saddlepoint to the vacuum gives $\left(g^{2} / 8 \pi^{2}\right) \int E_{i}^{a} B_{i}^{a} d^{3} x d \tau$ of almost exactly $\pm 1 / 2$.

This definition of $N_{\mathrm{CS}}$ has a somewhat arbitrary parameter $\tau_{0}$, so we cannot give a direct physical interpretation to the probability distribution of $N_{\mathrm{CS}}$. Also, the probability distribution of $N_{\mathrm{CS}}$ will depend on $\tau_{0}$. Consider a configuration which is "fairly close to" a sphaleron but which is already rolling away from the saddlepoint at cooling time $\tau_{0}$. If we increase $\tau_{0}$, it will roll further out of the saddlepoint before we start to integrate $E \cdot B$, and if we decrease $\tau_{0}$ then it will be closer to the saddlepoint. Hence, choosing a larger $\tau_{0}$ will spread out the determined values of $N_{\mathrm{CS}}$ of similar, nearly sphaleron configurations more widely, diluting the probability distribution near $N_{\mathrm{CS}}=1 / 2$ (and concentrating the probability distribution near $N_{\mathrm{CS}}=0$ ). This reduces the population of configurations with $N_{\mathrm{CS}}$ within $\epsilon$ of $1 / 2$. However, spreading out the configurations increases $d N_{\mathrm{CS}} / d t$, evaluated on an ensemble of configurations with $N=1 / 2$, by "almost" the same factor, and so the rate $\Gamma_{d}$ "almost" does not depend on $\tau_{0}$. By "almost" we mean that this statement is strictly true when we can neglect the contribution to $|d N / d t|$ arising from residual UV fluctuations. The more we cool, the larger the contribution to $d N / d t$ from motion in the unstable direction and the smaller the contributions from the stable directions, so the truer this becomes. In a complete calculation including the "dynamical prefactor" this remaining $\tau_{0}$ dependence is also accounted for [13]. For this work we will use a value of $\tau_{0}$ where we can directly check that fluctuations about the sphaleron are largely absent for configurations with $N_{\mathrm{CS}}=1 / 2$.

We make two further approximations to make measuring $N_{\mathrm{CS}}$ numerically practical. The cooling is very numerically expensive, but after cooling by $\tau>a^{2}$ all UV information on the lattice has been destroyed, and cooling on such a fine lattice is redundant. We set up a coarsened lattice of all even sites and continue the cooling on it. We have compared the value of $N_{\mathrm{CS}}$ obtained in this way to the value without coarsening, and aside from a slight and measurable renormalization, the difference is a tiny amount of noise. We also extrapolate the large $\tau$ exponential tail of $\int E \cdot B d \tau$ to avoid having to run $\tau$ to arbitrarily large times.

\section{Multicanonical measurement}

Now that we have a definition of $N_{\mathrm{CS}}$ (modulo 1), we want to measure the probability to have a particular value, that is

$$
P(x) \equiv \frac{1}{Z \delta x} \int \mathcal{D} \Phi \mathcal{D} A e^{-\beta H(\Phi, A)} \Theta\left(N_{\mathrm{CS}}-x\right) \Theta\left(x+\delta x-N_{\mathrm{CS}}\right),
$$

where $Z$ is the same integral without the $\Theta$ functions, which select out the range of $N_{\mathrm{CS}}$ between $x$ and $x+\delta x$. We are making the dimensional reduction approximation including the integration over the $A_{0}$ field, though we do include the $\mathrm{U}(1)$ hypercharge field at the physical value of $\Theta_{W}$ the Weinberg angle, using the noncompact lattice implementation.

To determine $P(x)$ accurately even where it is small, we perform a multicanonical MonteCarlo calculation. That is, we rewrite Eq. (8) as

$$
P(x) \equiv \frac{1}{Z \delta x} \int\left(\mathcal{D} \Phi \mathcal{D} A e^{-\beta H(\Phi, A)} e^{f\left(N_{\mathrm{CS}}\right)}\right) e^{-f\left(N_{\mathrm{CS}}\right)} \Theta\left(N_{\mathrm{CS}}-x\right) \Theta\left(x+\delta x-N_{\mathrm{CS}}\right),
$$



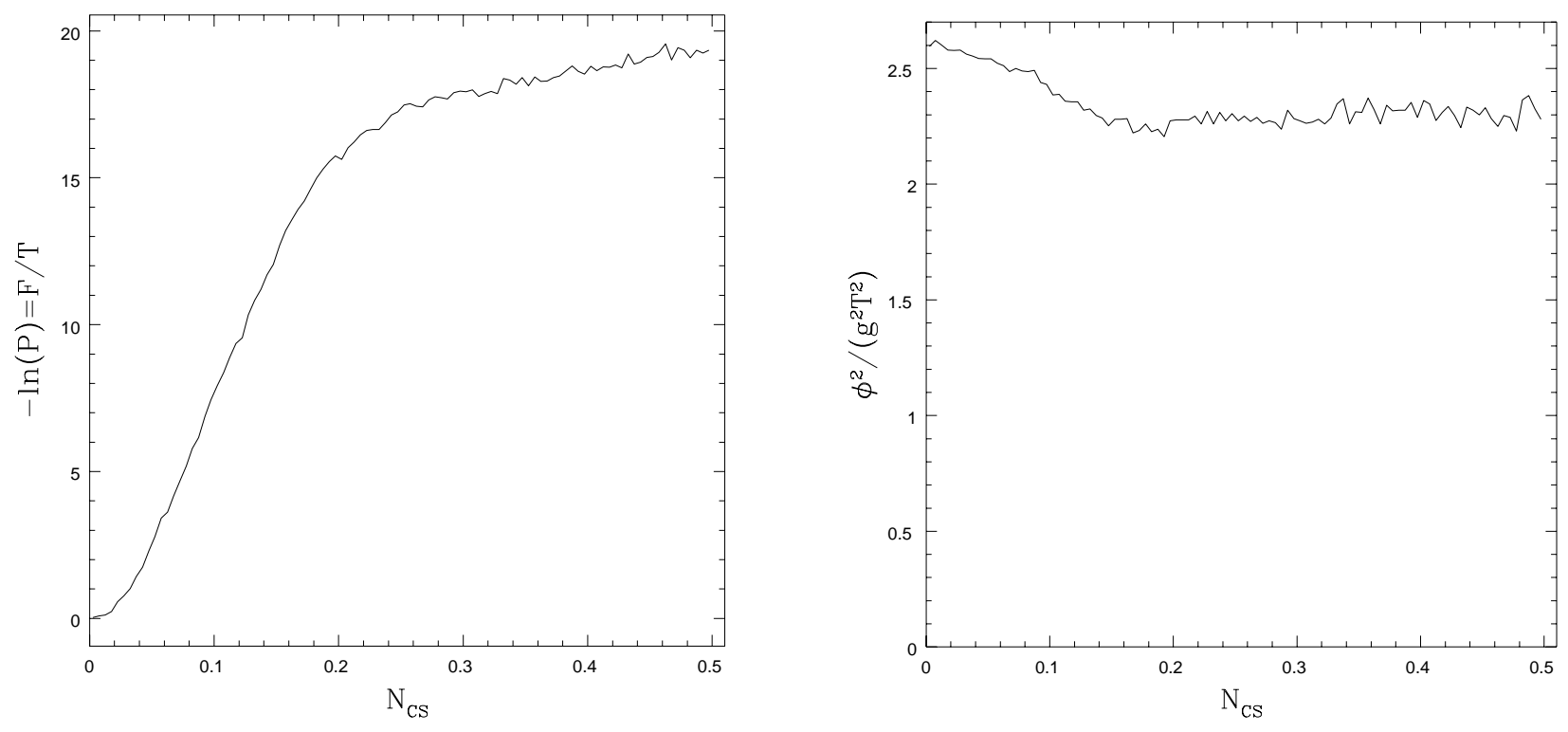

Figure 2: Free energy (left) and $\left(\phi_{\text {broken }}^{2}-\phi_{\text {symm }}^{2}\right) /\left(g^{2} T^{2}\right)$ as functions of $N_{\mathrm{CS}}$ at $x \equiv \lambda / g^{2}=$ 0.039 , in a $\left(16 / g^{2} T\right)^{3}$ volume. The plot of $\phi^{2}$ is a check that the volume used was large enough to prevent the sphaleron from causing a transition to the symmetric phase.

where $f(x)$ is a function chosen by bootstrapping to be approximately equal to $-\ln P(x)$. We do the integral by finding a Markovian process which samples configuration space with weight $e^{-\beta H(\Phi, A)} e^{f\left(N_{\mathrm{CS}}\right)} \mathcal{D} \Phi \mathcal{D} A$, and replacing the integral with a sum over a sample generated by that Markovian process. Since $N_{\mathrm{CS}}$ is a complicated function of the configuration, this is easiest done by finding a Markovian process which samples with canonical weight and adding a Metropolis accept reject step to account for $e^{f}$.

We generate the canonical Markovian process with the algorithm from [1], extending it to include the noncompact $\mathrm{U}(1)$ subgroup, and reducing the efficiency of the gauge field updates, to bring the Metropolis accept rate up to about $50 \%$. To measure $\left|d N_{\mathrm{CS}} / d t\right|$, we augment the system with Gaussian "momentum" degrees of freedom, pick momenta from the thermal distribution, and evolve all fields forward under Hamilton's equations (implemented by leapfrog) for a (very short) time. We determine $d N_{\mathrm{CS}} / d t$ from the difference between $N_{\text {CS }}$ of the initial and final configurations. This differs from a Hamiltonian evolution of the classical system because we do not enforce Gauss' law; but since $B$ is transverse and Gauss' law only affects the longitudinal $E$ field, the instantaneous value of $E \cdot B$ should not be affected.

We have measured $\Gamma_{d}$ for three values of scalar self-coupling, $x \equiv\left(\lambda / g^{2}\right)=0.047,0.039$, and 0.033 . We used a $40^{3}$ box with periodic boundary conditions and a lattice spacing of $a=2 /\left(5 g^{2} T\right)\left(\beta_{\mathrm{L}}=10\right)$ for the larger values of $x$ and $a=1 /\left(3 g^{2} T\right)\left(\beta_{\mathrm{L}}=12\right)$ for $x=0.033$. We determine the equilibrium temperature as in [20]. The lattice action is $O(a)$ improved using the relations in [21, 22], so finite lattice spacing systematic errors will be smaller than statistical errors. The very large volume is necessary to prevent the tails of the sphaleron from meeting because of the periodic boundary conditions. It is also necessary because the Higgs field condensate has a zero at the core of a sphaleron, so the sphaleron looks somewhat 


\begin{tabular}{|cc|c|c|c|}
\hline & & $x=0.047$ & $x=0.039$ & $x=0.033$ \\
\hline \multirow{3}{*}{$\begin{array}{c}\text { "2 loop" } \\
\text { perturbative }\end{array}$} & $\phi\left(T_{\mathrm{c}}\right) / g T_{\mathrm{c}}$ & 1.360 & 1.568 & 1.789 \\
\cline { 2 - 5 } & $B \equiv g E_{\mathrm{sph}} / 4 \pi \phi$ & 1.643 & 1.633 & 1.626 \\
\cline { 2 - 5 } & $E_{\mathrm{sph} / T_{\mathrm{c}}}$ & 28.08 & 32.20 & 36.55 \\
\cline { 2 - 5 } & $-\ln \left(\Gamma_{d} T_{\mathrm{c}}^{-4}\right)$ & 22.27 & 25.39 & 28.82 \\
\hline \multirow{2}{*}{ nonperturbative } & $-d\left(\Gamma_{d} T_{\mathrm{c}}^{-4}\right) / d y$ & 860 & 920 & 1000 \\
\cline { 2 - 5 } & $\phi\left(T_{\mathrm{c}}\right) / g T_{\mathrm{c}}$ & $1.38 \pm 0.02$ & $1.60 \pm 0.01$ & $1.82 \pm 0.03$ \\
\hline mixed & $-\ln \left(\Gamma_{d} T_{\mathrm{c}}^{-4}\right)$ & $24.7 \pm 0.4$ & $28.3 \pm 0.4$ & $31.2 \pm 0.6$ \\
\hline
\end{tabular}

Table 1: Perturbation theory versus nonperturbative $\Gamma_{d}$. Appearances of $T_{\mathrm{c}}^{-4}$ are really $\left(2.5 g^{2} T_{\mathrm{c}}\right)^{-4}$, and $d / d y$ means $g_{w}^{4} d / d\left(m_{\mathrm{H}}^{2} / T^{2}\right)$. The error bars for the nonperturbative $\phi\left(T_{\mathrm{c}}\right)$ are dominated by statistical errors in the determined value of $T_{c}$; errors in the nonperturbative value of $\Gamma$ are statistical errors from the Monte-Carlo. The "mixed" results use the two loop value of $B$ but the nonperturbative value of $\phi\left(T_{\mathrm{c}}\right)$.

like a symmetric phase bubble; the large volume prevents the sphaleron from stimulating a transition to the symmetric phase.

We plot the numerically determined free energy and $\left\langle\phi^{2}\right\rangle$ versus $N_{\mathrm{CS}}$ for the $x=0.039$ data in Fig. 2. The value of $\left\langle\left|d N_{\mathrm{CS}} / d t\right|\right\rangle$ at $N_{\mathrm{CS}} \simeq 0.5$ was $(0.22 \pm 0.05) a^{-1}=(0.55 \pm 0.13) g^{2} T$. Recall that both this value, and free energy distribution, depend somewhat on our choice of $\tau_{0}$, which was $\tau_{0}=22.5 a^{2}=3.6 / g^{4} T^{2}$. With less cooling, the free energy would rise more evenly and would be less flat on top; so the shape of the free energy curve should not be overinterpreted. The determined rate $\Gamma_{d}$ should be independent of $\tau_{0}$, though.

We present the results in Table 11. They should be compared to the symmetric phase rate, which, using $g^{2}=0.40$, is $\Gamma_{d}=(29 \pm 6) \alpha_{W}^{5} T^{4}=\exp (-13.9 \pm 0.2) T^{4}$ [12 $]^{3}$. As expected, the rate is orders of magnitude smaller in the broken phase than in the symmetric phase.

\section{Comparison to perturbation theory, erasure bound}

Next we compare the rate to a perturbative estimate. One loop perturbation theory gives [5]田

$$
\Gamma_{d}=4 T^{4} \frac{\omega_{-}}{g \phi}\left(\frac{\alpha_{W}}{4 \pi}\right)^{4}\left(\frac{4 \pi \phi}{g T}\right)^{7} \mathcal{N}_{\mathrm{tr}} \mathcal{N} \mathcal{V}_{\mathrm{rot}} \kappa e^{-\beta E_{\mathrm{sph}}}
$$

Here $\phi$ is the broken phase Higgs condensate expectation value, $\omega_{-}$is the unstable frequency of the sphaleron, $\mathcal{N}_{\text {tr }} \mathcal{N} \mathcal{V}_{\text {rot }}$ are zero mode factors, $\kappa$ is the one loop fluctuation determinant, and $E_{\mathrm{sph}}$ is the energy of the Klinkhamer Manton sphaleron, using the tree level Hamiltonian. For small scalar self-coupling, $-T \ln \kappa$ equals the energy due to the one loop effective potential term, plus a modest correction [25]. We can guess that the dominant two loop corrections to Eq. (10) are absorbed by including the two loop effective potential terms in the Hamiltonian.

\footnotetext{
${ }^{3}$ Again, this result misses a logarithmic correction which is computable but not computed [14.

${ }^{4}$ The definition of $\Gamma$ used in [5, 23] is the response rate to a chemical potential, which is half the diffusion rate [24]; so Eq. (10) differs by a factor of 2 from the expressions in those references.
} 
So it seems reasonable to estimate the sphaleron rate by Eq. (10), but setting $\kappa=1$ and solving for the sphaleron energy using the two loop effective potential at the equilibrium temperature. One should also solve for the zero modes and $\omega_{-} / \phi$ at this value, but they are very weak functions of the effective potential [23]. We use the values from [23] at $x=\left(\lambda / g^{2}\right)=0.04$ for these, but solve for the sphaleron energy, $E_{\mathrm{sph}}=4 \pi B \phi / \mathrm{g}$, numerically, using the two loop effective potential at $T_{\mathrm{c}}$. We use the two loop potential presented in [26], without pieces from longitudinal gauge bosons (assumed integrated out). We also drop two loop terms proportional to $\lambda g^{2}$ or $\lambda^{2}$, because the perturbative determination of $\phi$ is an expansion in $\lambda / g^{2}$, and such terms contribute at the same or higher order as unknown 3 loop terms. (Including those terms moves $\phi$ closer to the nonperturbative value.) We compare the results to the numerically determined values in Table 1. The "two loop" analytic sphaleron rate is about $\exp (2.5)$ times faster than the numerically determined nonperturbative rate. The difference is more than can be explained by the difference in $\phi$.

We should compare the sphaleron rate to the limit set by requiring that baryon number not be erased. The rate at which sphalerons degrade baryon number is [5]

$$
\frac{1}{N_{\mathrm{B}}} \frac{d N_{\mathrm{B}}}{d t}=-\frac{13 N_{\mathrm{F}}}{4} \Gamma_{d} T^{-3}
$$

where $N_{\mathrm{F}}=3$ is the number of families, and the numerical factor $13 N_{\mathrm{F}} / 4$ would be smaller in theories, such as supersymmetry, in which additional degrees of freedom can store baryon number.f Integrating from the end of the phase transition to the present day,

$$
\ln \left(N_{\mathrm{B}} / N_{\mathrm{B}}\left(T_{\mathrm{c}}\right)\right)=-\frac{13 N_{\mathrm{F}}}{4} \int_{t_{0}}^{\infty} \Gamma_{d}(T(t)) T^{-3}(t) d t
$$

where we have shown the dependence of $\Gamma_{d}$ on $T$ and of $T$ on $t$.

Now $\ln \Gamma_{d}$ is very sensitive to $\sqrt{\left\langle\phi^{2}\right\rangle}$, and hence to $T$; so we can approximate $\ln \Gamma_{d}(T) \simeq$ $\ln \Gamma_{d}\left(T_{\mathrm{c}}\right)+\left.\left(T-T_{\mathrm{c}}\right)\left(d \ln \Gamma_{d} / d T\right)\right|_{T=T_{\mathrm{c}}}$, and perform the integral:

$$
\ln \left(-\ln \left(N_{\mathrm{B}} / N_{\mathrm{B}}\left(T_{\mathrm{c}}\right)\right)\right)=\ln (39 / 4)+\ln \left(\frac{\Gamma_{d}\left(T_{\mathrm{c}}\right)}{T^{4}}\right)-\ln \left(-\left.\frac{d \ln \Gamma_{d}(T(t))}{T d t}\right|_{T=T_{\mathrm{c}}}\right) .
$$

By the chain rule,

$$
\frac{d \ln \Gamma_{d}}{T d t}=\frac{d \ln \Gamma_{d}}{d y} \frac{d y}{d T} \frac{d \ln T}{d t},
$$

where $y=m_{\mathrm{H}}^{2}(T) /\left(g^{4} T^{2}\right)$ is the dimensionless thermal Higgs mass squared.

Two of these are easy. We get $d y / d T$ from the 1 loop correction to $m_{\mathrm{H}}^{2}$ [26],

$$
\frac{d y}{d T} \simeq \frac{8 \lambda+4 g_{\mathrm{y}}^{2}+g^{2}\left(3+\tan ^{2} \Theta_{W}\right)}{8 g^{4} T}
$$

and we get $d \ln T / d t$ from the Friedmann equation in a radiation dominated universe,

$$
\frac{1}{4 t^{2}}=H^{2}=\frac{8 \pi G}{3} \frac{\pi^{2} g_{*}}{30} T^{4} \Rightarrow \frac{d \ln T}{d t}=-\sqrt{\frac{4 \pi^{3} g_{*}}{45}} \frac{T^{2}}{m_{\mathrm{pl}}},
$$

\footnotetext{
${ }^{5}$ Again, there is a factor of 2 difference from the reference because they write in terms of the response to a chemical potential, which is half the diffusion constant.
} 
where $g_{*}$ is the number of radiative degees of freedom in the universe $\left(g_{*}=106.75\right.$ in the minimal standard model) and $m_{\mathrm{pl}} \simeq 1.22 \times 10^{19} \mathrm{GeV}$ is the Planck mass. Finally, we determine $d \ln \Gamma_{d} / d y$ perturbatively, by varying $y$ slightly from the equilibrium value and recomputing the two loop sphaleron rate. The dependence is quite strong. We include it in the table.

The most widely cited discussion of baryon number erasure after the phase transition makes the approximation that the baryon number violation rate after the phase transition is constant for about one Hubble time [27]. In fact, because $\Gamma_{d}$ depends very strongly on $y$, which in turn depends strongly on $T$, most baryon number erasure occurs in the first $10^{-3}$ Hubble times after the phase transition. Hence the initial rate of baryon number violation, $\Gamma_{d}\left(T_{\mathrm{c}}\right)$, which prevents washout is $10^{3}$ times larger than assumed in [27, leading to a weaker bound on $\Gamma_{d}\left(T_{\mathrm{c}}\right)$, roughly

$$
-\ln \left(\Gamma_{d}\left(T_{\mathrm{c}}\right) T_{\mathrm{c}}^{-4}\right)>30.4-\ln \left(T_{\mathrm{c}} / 100 \mathrm{GeV}\right) .
$$

The values of $g_{*}$ and $d y / d T$ are both larger in supersymmetric extensions of the standard model, by on order a factor of 2; so the bound, Eq. (17), is weaker in those models by about 1. Also note that, because Eq. (13) is for the double log of $N_{\mathrm{B}} / N_{\mathrm{B}}\left(T_{\mathrm{c}}\right)$, failing to meet the bound by 1 means the baryon number is diminished by $\exp (\exp (1)) \simeq 15$, and failure by 2 reduces baryon number by $\exp (\exp (2)) \simeq 1600$; so the bound is quite sharp.

Interpolating between the values of $x$ where we have measured, and including the estimate discussed in the conclusion for the dynamical prefactor, we get a bound of about $x=0.036$ in the standard model and $x=0.038$ in the MSSM.

\section{Conclusion}

Our nonperturbative results show that the bound on $x \equiv \lambda / g^{2}$ is about 0.036 in the standard model. This result will be corrected only slightly in any theory where all non-standard model degrees of freedom are heavy enough to integrate out accurately. This should apply for instance to the minimal supersymmetric standard model unless the lightest stop is quite light [16]. The bound weakens somewhat if the latent heat of the phase transition is not sufficient to reheat the universe to $T_{\mathrm{c}}$; this should be checked in the MSSM.

If additional degrees of freedom are light, then they can affect the thermodynamics of the sphaleron (aside from renormalizing couplings). The calculation should then be redone, including those degrees of freedom. However, we have shown that if a two loop perturbative calculation gets the value of $\phi$ about right, then its value for $\Gamma_{d}$ is reasonably close. We do not expect light stop squarks to endanger this conclusion; while their contribution to the effective potential may be important, they only contribute to the scalar wave function at two loops, and they do not interact directly with the gauge fields at all. Hence, they should change the sphaleron bound mainly by changing the size of the Higgs condensate. We can paraphrase the results of this work as setting a bound on $\phi$ of roughly $\phi \geq 1.7 g T$, and this should be approximately the same in extensions with a light stop.

Finally we should comment on the approximation that successive sphaleron crossings will be in uncorrelated directions. Arnold, Son, and Yaffe have recently criticized this assumption [28], on the grounds that it ignores dynamical effects related to hard thermal loops. Their 
picture has recently been borne out in the symmetric phase [12]. In the present context, we would expect the system to cross the sphaleron barrier multiple times before relaxing to a vacuum configuration if the plasma frequency $\omega_{\mathrm{pl}}$ is much greater than the unstable mode frequency $\omega_{-}$. It should be clear why; when $\omega_{\mathrm{pl}} \gg \omega_{-}$, the electric field, and hence $E_{i}^{a} B_{i}^{a}$, will oscillate in sign, driving $N_{\mathrm{CS}}$ back and forth, on a time scale short compared to the time scale at which it "falls off" the sphaleron. On the other hand, if $\omega_{\mathrm{pl}}<\omega_{-}$, then we expect no such effect. A crude, parametrically correct estimate is that the rate we determined will be corrected by a factor of

$$
\frac{1}{1+\omega_{\mathrm{pl}}^{2} / \omega_{-}^{2}}=\left[1+\left(\frac{11 g^{2} T^{2}}{18}\right)\left(0.47 g^{2} \phi^{2}\right)^{-1}\right]^{-1} .
$$

Here we use the standard model value for $\omega_{\mathrm{pl}}^{2}$ and the value of $\omega_{-}^{2}$ computed in [23] for $\lambda \simeq 0.03 g^{2}$. Numerically, using $g^{2} \simeq 0.4$, this estimate reduces the rate by a factor of 2 for $x=0.033$, changing the bound on $x=\lambda / g^{2}$ by $\simeq 0.0015$. The correction in the symmetric phase is larger, because the natural length scale of a winding number changing process is larger; if we estimate that scale as $g^{2} T / 2$, for instance, the correction would be $\sim 7$. It is possible to use the techniques of [12] to measure the prefactor numerically [13], but we will not do so here.

\section{Acknowledgments}

I would like to thank Jim Cline, Jim Hetrick, Kari Rummukainen, and Alex Krasnitz for discussions and correspondence. I also thank Berndt Müller and the North Carolina Supercomputing Center.

\section{References}

[1] K. Kajantie, M. Laine, K. Rummukainen, and M. Shaposhnikov, Nucl. Phys. B 466, 189 (1996); Phys. Rev. Lett. 77, 2887 (1996).

[2] K. Kajantie, M. Laine, K. Rummukainen, and M. Shaposhnikov, Nucl. Phys. B 493, 413 (1997).

[3] H. Dosch, J. Kripfganz, A. Laser, and M. Schmidt, Phys. Lett. B 365, 213 (1996); M. Gurtler, E. Ilgenfritz, J. Kripfganz, H. Perlt, and A. Schiller, Nucl. Phys. B 483, 383 (1997); M. Gurtler, E. Ilgenfritz, and A. Schiller, hep-lat/9702020; hep-lat/9704013.

[4] M. Gavela, P. Hernandez, J. Orloff, and O. Pene, Nucl. Phys. B 430 (1994) 345; M. Gavela, P. Hernandez, J. Orloff, O. Pene, and C. Quimbay, Nucl. Phys. B 430 (1994) 382.

[5] P. Arnold and L. McLerran, Phys. Rev. D 36, 581 (1987).

[6] G. t'Hooft, Phys. Rev. Lett. 37,8 (1976). 
[7] F. Klinkhamer and N. Manton, Phys. Rev. D 30, 2212 (1984).

[8] J. Langer, Ann. Phys. (N. Y.) 41 (1967) 108; ibid 54 (1969) 258.

[9] I. Affleck, Phys. Rev. Lett. 46 (1981) 388.

[10] G. D. Moore and N. Turok, PUPT-1681, hep-ph/9703266.

[11] J. Ambjørn and A. Krasnitz, hep-ph/9705380.

[12] G. D. Moore, C. Hu, and B. Müller, hep-ph/9710436.

[13] G. D. Moore, MCGILL-98/17, hep-ph/9805264.

[14] D. Bödeker, Phys. Lett. B 426, 351 (1998).

[15] G. D. Moore, in preparation.

[16] J. Cline and K. Kainulainen, Nucl. Phys. B 482 (1996) 73; hep-ph/9705201; M. Losada, Phys. Rev. D 56 (1997) 2893; M. Losada and G. Farrar, Phys. Lett. B 406 (1997) 60; M. Losada, hep-ph/9612337; M. Laine, Nucl. Phys. B 481 (1996) 43.

[17] J. Hetrick and Ph. de Forcrand, hep-lat/9710003.

[18] J. Ambjørn and A. Krasnitz, Phys. Lett. B 362, 97 (1995).

[19] G. D. Moore, Nucl. Phys. B 480, 657 (1996).

[20] F. Csikor, Z. Fodor, J. Hein, and J. Heitger, Phys. Lett. B 357 (1995) 156.

[21] G. D. Moore, Nucl. Phys. B 493, 439 (1997).

[22] G. D. Moore, hep-lat/9709053.

[23] L. Carson and L. McLerran, Phys. Rev. D 41 (1990) 647.

[24] S. Khlebnikov and M. Shaposhnikov, Nucl. Phys. B308, 885 (1988).

[25] J. Baacke and S. Junker, Phys. Rev. D 49 (1994) 2055; Phys. Rev. D 50 (1994) 4227.

[26] Z. Fodor and A. Hebecker, Nucl. Phys. B 432 (1994) 127.

[27] M. Shaposhnikov, Nucl. Phys. B 287 (1987) 757.

[28] P. Arnold, D. Son, and L. Yaffe, Phys. Rev. D 55, 6264 (1997). 